\title{
Follow-up study on serum cholesterol profiles and potential sequelae in recovered COVID-19 patients
}

Guiling $\mathrm{Li}^{1 \dagger}$, Li Dư ${ }^{2 \dagger}$, Xiaoling Cao ${ }^{3}$, Xiuqi Wei ${ }^{4}$, Yao Jiang ${ }^{1}$, Yuqi Lin ${ }^{4}$, Vi Nguyen ${ }^{3}$, Wenbin Tan $^{3,5^{*+}}$ and Hui Wang ${ }^{6^{*+}}$

\begin{abstract}
Background: COVID-19 patients develop hypolipidemia. However, it is unknown whether lipid levels have improved and there are potential sequlae in recovered patients.

Objective: In this follow-up study, we evaluated serum lipidemia and various physiopathological laboratory values in recovered patients.

Methods: A 3-6 month follow-up study was performed between June 15 and September 3, 2020, to examine serum levels of laboratory values in 107 discharged COVID-19 patients (mild = 59; severe/critical = 48; diagnoses on admission). Sixty-one patients had a revisit chest CT scan. A Wilcoxon signed-rank test was used to analyze changes in laboratory values at admission and follow-up.

Results: LDL-C and HDL-c levels were significantly higher at follow-up than at admission in severe/critical cases $(p<0.05)$. LDL-c levels were significantly higher at follow-up than at admission in mild cases $(p<0.05)$. Coagulation and liver functional values were significantly improved at follow-up than at admission for patients $(p<0.05)$. Increases in HDL-c significantly correlated with increases in numbers of white blood cells $(p<0.001)$ during patients' recovery. With exclusion of the subjects taking traditional Chinese medicines or cholesterollowering drugs, LDL-c and HDL-c levels were significantly increased at follow-up than at admission in severe/critical cases $(p<0.05$ ). Residue lesions were observed in CT images in $72 \%$ (44 of 61 ) of follow-up patients.

(Continued on next page)
\end{abstract}

\footnotetext{
*Correspondence: wenbin.tan@uscmed.sc.edu; m18971574342@163.com

${ }^{\dagger}$ Guiling Li, Li Du, Wenbin Tan and Hui Wang contributed equally to this work.

${ }^{3}$ Department of Cell Biology and Anatomy, School of Medicine, University of South Carolina, Columbia, SC 29209, USA

${ }^{6}$ Department of Laboratory Medicine, Union Hospital, Tongji Medical College, Huazhong University of Science and Technology, Wuhan 430022, People's Republic of China

Full list of author information is available at the end of the article
}

(C) The Author(s). 2021 Open Access This article is licensed under a Creative Commons Attribution 4.0 International License, which permits use, sharing, adaptation, distribution and reproduction in any medium or format, as long as you give appropriate credit to the original author(s) and the source, provide a link to the Creative Commons licence, and indicate if changes were made. The images or other third party material in this article are included in the article's Creative Commons licence, unless indicated otherwise in a credit line to the material. If material is not included in the article's Creative Commons licence and your intended use is not permitted by statutory regulation or exceeds the permitted use, you will need to obtain permission directly from the copyright holder. To view a copy of this licence, visit http://creativecommons.org/licenses/by/4.0/ The Creative Commons Public Domain Dedication waiver (http://creativecommons.org/publicdomain/zero/1.0/) applies to the data made available in this article, unless otherwise stated in a credit line to the data. 
(Continued from previous page)

Conclusions: Improvements of LDL-C, HDL-c, liver functions, and incomplete resolution of lung lesions were observed at 3-6 month follow-up for recovered patients, indicating that a long-term recovery process could be required and the development of sequelae such as pulmonary fibrosis could be expected in some patients.

Keywords: LDL-c, HDL-c, COVID-19, Cholesterol, Follow-up, CT, Residue lesions

\section{Introduction}

Coronavirus disease 2019 (COVID-19), which has become a major threat to the global public health system [1], is caused by severe acute respiratory syndrome coronavirus 2 (SARS-Cov-2) [2]. As of February 18, 2021, more than 110 million COVID-19 infections and 2.5 million deaths have been reported in 192 countries and regions. China reported 100 thousands cases and 4833 deaths which were mainly from the original epicenter, Wuhan [3]. Scientists are just beginning to understand the nature of the harm caused by this disease. The SARS-Cov- 2 spike protein mediates the entrance of the virus into host cells via surface angiotensin-converting enzyme 2 (ACE2) [4, 5]. Host protease transmembrane, serine protease 2 (TMPRSS2) promotes SARS-CoV-2 entry into target cells, which are thought to be host determinants for viral infection in the initial stage. COVID-19 patients may be asymptomatic or symptomatic. The time from exposure to onset of symptoms is about 5.1 days [6]. Pathologically, almost every vital organ in the body, including the lungs, heart, liver, kidneys, eyes, blood vessels, intestines, and brain, can be injured by SARS-CoV-2, leading to devastating consequences [7]. Acute and diffuse lung injuries cause increases in a series of serum cancer biomarkers in patients [8]. Damage to the lungs can be long-term for some recovered patients; this has also been observed in surviving severe acute respiratory syndrome (SARS) patients [9].

Patients with metabolic-associated preconditions are susceptible to SARS-CoV-2 attack and are likely to experience more pronounced symptoms. One pathogenic cofactor associated with hypertension, obesity, diabetes mellitus, and cardiovascular disorders is hypercholesterolemia. We and others have recently reported hypolipidemia in hospitalized COVID-19 patients [10-13]. An association between decreases in lipid levels and the severity of the symptoms in patients has been revealed [10-12]. Furthermore, emerging evidence has shown that SARS-CoV-2 has a direct impact on the downregulation of lipid-metabolismrelated proteins and pathways, leading to dyslipidemia [14]. In addition, dyslipidemia associated with SARS-CoV-1 has also been reported [15]. Altered lipid metabolism has been shown in recovered SARS-CoV-1 patients 12 years after infection [16]. These reports demonstrate that dyslipidemia is an important clinical manifestation in patients with coronavirus-related diseases, perhaps reflecting one aspect of the complicated evolving pathological progressions seen in patients. In this study, we performed a follow-up investigation of lipid profiles and other laboratory values in COVID-19 patients from our previously reported cohort [17]. We found that there were significant improvements of coagulation and liver laboratory values, including D-dimer, antithrombin III (ATIII), fibrin degradation product (FDP), fibrinogen (FIB), alanine aminotransferase (ALT), alkaline phosphatase (ALP), and gamma-glutamyl transferase (GGT) in patients at follow-up compared to time of admission. However, partial recovery of low-density lipoprotein cholesterol (LDL-c) levels and incomplete absorption of lung lesions were observed at follow-up, indicating that a long-term recovery process and the development of sequelae such as pulmonary fibrosis could be expected in some patients.

\section{Methods}

\section{Study design and patients}

All methods in this study were performed in accordance with the relevant guidelines and regulations. This follow-up study was carried out at the Cancer Center at the Union Hospital of Tongji Medical College, Huazhong University of Science and Technology in Wuhan, P. R. China, and was approved by the Institutional Review Board (IRB) at the Union Hospital of Tongji Medical College, Huazhong University of Science and Technology in Wuhan, P. R. China. The need for written informed consent was waived by the IRB committee at the Union Hospital of Tongji Medical College, Huazhong University of Science and Technology in Wuhan, P. R. China. Our previous study included a cohort of 597 COVID-19 patients admitted to the hospital between February 1 and March 3, 2020 [11]. In June and August, 2020, we attempted phone inquiries to all patients in this cohort; we successfully reached 260 patients and received informed consents over the phone from 144 of them to take follow-up laboratory tests. Ultimately, a total of 97 patients participated the follow-up study on June 2020 and 10 patients participated the follow-up study on September 2020. In order to compare laboratory values at admission with those at follow-up, the patients were grouped into the same categories assigned to them in the original study, which were based on their diagnoses on admission [11], that is, mild $(n=59)$ and severe/critical $(n=48)$ cases. Briefly, clinical diagnostic criteria and guideline were as 


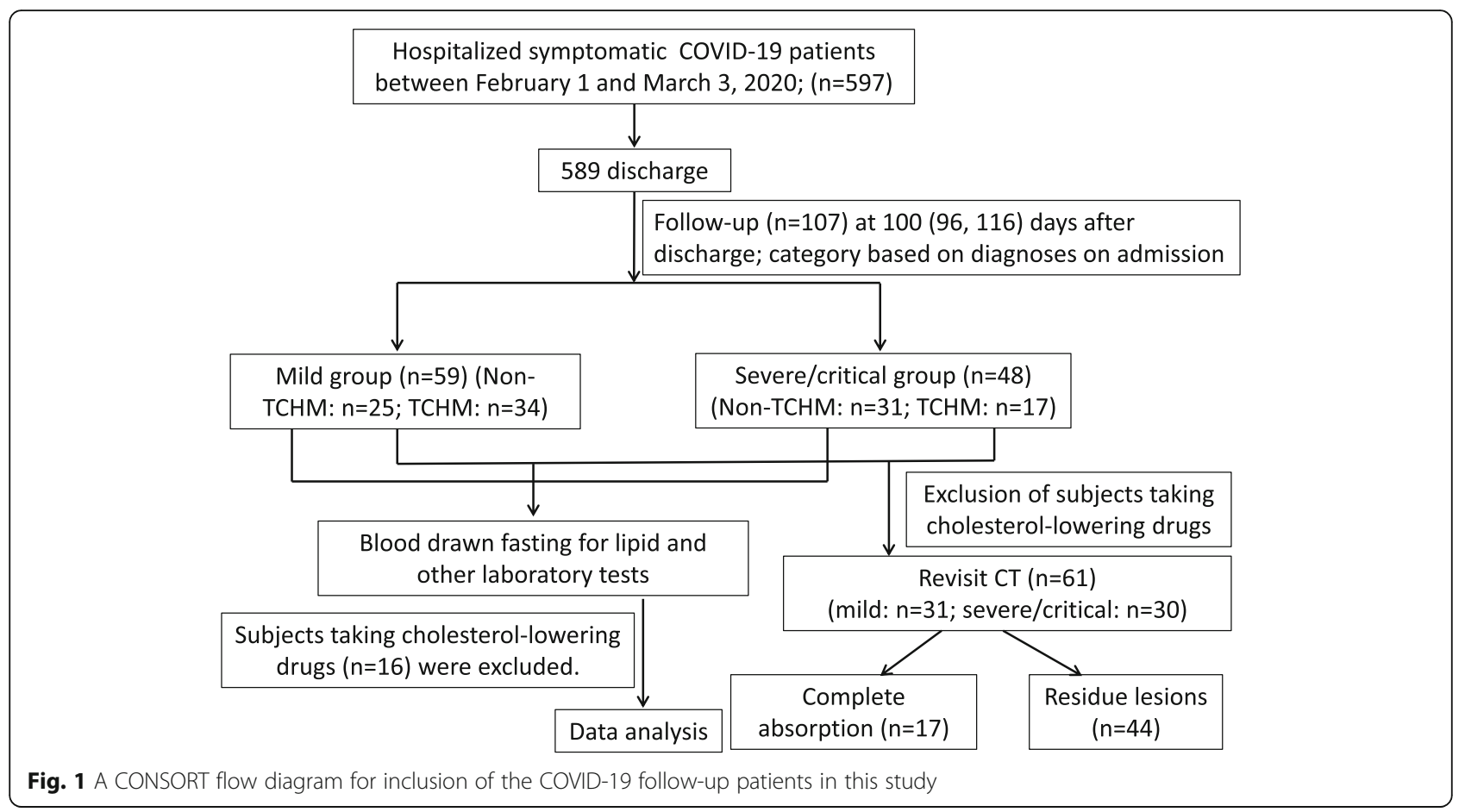

the follows: (1) mild group, patients with onset of symptoms including fever, cough, fatigue, headache, diarrhea, and so forth, with or without mild pneumonia; (2) severe group, patients showed dyspnea, acute respiratory stress, decrease in blood oxygen saturation, lung infiltrates, multiple peripheral ground-glass opacities on both lungs; and (3) critical group, patients presented respiratory or multiple organ failure and septic shock [11]. A revisit CT scan was performed on a total of 61 patients as their standard care of follow-up examination which was covered by their health insurance providers. A CONSORT flow diagram is shown in Fig. 1.

Many patients were prescribed traditional Chinese herbal medications (TCHM) by their local healthcare workers as a standard supplementary treatment of COVID-19 in Wuhan. Two typical TCHMs were widely given to patients with mild symptoms or at the onset of symptoms or/and after discharge from hospitals for one or 2 weeks according to Chinese Center for Disease Control (CDC) guidelines. These TCHMs were believed to be able to suppress inflammatory responses and improve lung functions in patients. To adjust the factor of THCM for a further analysis, we categorized patients into non-TCHM or TCHM subgroup to assess the potential effects of these THCMs on patients' lipid profiles.

\section{Clinical laboratory tests and CT image acquisition}

White blood cell (WBC), lymphocyte (LY), and monocyte (MO) counts were performed on a Beckman LH750 analyzer using the manufacturer's reagents (Beckman
Coulter, Brea, California, USA). A Beckman AU5800 chemistry analyzer (Beckman Coulter, Brea, CA, USA) was used for tests for the following laboratory values: ALT, ALP, GGT, LDL-c, high-density lipoprotein cholesterol (HDL-c), total cholesterol (TC), triglycerides (TG), total protein (TP), albumin (ALB), globulin (GLB), total bilirubin (TBIL), direct bilirubin (DBIL), and total bile acid (TBA). LDL-c, HDL-c, and TC were determined with standard homogeneous assays from Beckman Coulter (Catalog Nos. OSR6283, OSR6587, and OSR6616). D-dimer, ATIII, FDP, and FIB were tested on a Stago STAR analyzer using the manufacturer's reagents (Stago, Parsippany, New Jersey, USA). C-reactive protein (CRP) was determined using a BC-5390 analyzer (Mindray, Shenzhen, Guangzhou, P. R. China). The clinical laboratory data included in this study were from blood samples drawn fasting on the morning of the day of follow-up, a standard time and procedure of blood drawn for laboratory tests in our hospital including blood sample collections at admission in our previous study [11]. The blood samples were taken from patients in a sitting position at both admission and follow-up. Electronic data from the time of the patients' hospitalization, including demographic information, clinical symptoms and diagnosis, laboratory tests, and treatment data, were also extracted for comparison. The ratio of laboratory index was calculated using the values at follow-up to divide those on admission.

CT scanners (GE LightSpeed 16, GE VCT LightSpeed 64 from GE Healthcare, Chicago, Illinois, USA) were 
used to take chest CT scans with the following parameters: tube voltage $=120 \mathrm{kVp}$; current intelligent control $=$ $30-300 \mathrm{~mA}$; and slice thickness $/$ interval $=0.6-1.5 \mathrm{~mm}$. The following typical abnormal patterns for viral pneumonia were reported in COVID-19 CT images [18]: ground glass opacities (GGO), consolidation/nodules, and shadows.

\section{Statistical analysis}

Statistical analyses were performed with the statistical software SPSS (IBM, Armonk, New York, USA). Data are presented as mean (SD) or median (interquartile range, IQR). A Wilcoxon signed-rank test (two-sided) was used to compare patient laboratory values at admission and follow-up. Mann-Whitney U test was used to compare differences of lipid values at the time of admission or follow-up in the non-TCHM subgroup with TCHM subgroup. A Spearman correlation analysis was used to calculate correlation coefficiency. $P$ values of $p<0.05$ were considered as statistically significant.

\section{Results}

\section{Demographic and clinical characteristics of COVID-19} patients

A total of 107 COVID-19 cases were included in this follow-up study: 59 mild and 48 severe/critical cases (based on patient diagnosis at time of admission). A CONSORT flow diagram is shown in Fig. 1. The age for all patients was $65(60,70)$ (median (IQR)) years. The ages for patients in the two subgroups were as follows: $65(51,69)$ for mild and $66(62,74)$ for severe/critical cases (Table 1). The overall follow-up time was 100 (96, 116) days after discharge. The ratios for comorbidities, days until follow-up, and sex disparity among patients in each category are listed in Table 1. A small percentage

Table 1 Demographic and clinical characteristics of follow-up COVID-19 patients

\begin{tabular}{llll}
\hline Characteristics & \multicolumn{2}{l}{ COVID-19 follow-up patients } & $P$ \\
\cline { 2 - 3 } Category $(\boldsymbol{n})$ & Mild (59) & Severe/Critical (48) & \\
\hline Age, years & $65(51,69)$ & $66(62,74)$ & n.s. \\
Male & $28(47 \%)$ & $25(52 \%)$ & n.s. \\
Female & $31(53 \%)$ & $23(48 \%)$ & n.s. \\
Comorbidities & & & \\
$\quad 2-D M$ & $6(10 \%)$ & $6(12 \%)$ & n.s. \\
$\quad$ Hypertension & $21(36 \%)$ & $23(48 \%)$ & n.s. \\
$\quad$ CVD & $6(10 \%)$ & $8(16 \%)$ & n.s. \\
Gastrointestinal diseases & $4(7 \%)$ & $2(4 \%)$ & n.s. \\
Follow-up (days) & $93(82,96)$ & $101(96,118)$ & n.s. \\
Cholesterol-lowering drugs & $9(15 \%)$ & $7(18 \%)$ & n.s. \\
\hline
\end{tabular}

Data are presented as median (IQR) or $n$ (\%); n.s. no significance, CVD cardiovascular disease, and 2-DM = type-2 diabetes mellitus. Chi-Square test is used of patients in the subgroups (about 15-18\%) used cholesterol-lowering drugs (Table 1).

\section{Improvements of serum cholesterol levels in recovered COVID-19 patients}

Our previous studies have shown that serum LDL-c, HDL-c and TC levels at the time of admission were significantly lower in COVID-19 patients than in normal subjects $[10,11]$. In this follow-up study, we compared patient lipid levels at 3-6 months after discharge to those at the time of admission. Both LDL-c, HDL-c and TC were significantly higher at follow-up than at the time of admission in severe / critical cases (Table 2). LDL-c and TC levels were significantly higher at follow-up than at the time of admission in mild patients (Table 2). Surprisingly, after exclusion of those taking cholesterollowering drugs, $6 \%$ of patients (6 of 91) showed a $15 \%$ or more decrease in LDL-c levels at follow-up as compared to the time of admission; these patients included 3 mild and 3 severe/critical cases. In addition, the same percentages of patients $(6 \%, 6$ of 91$)$ showed a $15 \%$ or more decrease in HDL-c levels at follow-up as compared to the time of admission; these patients included 4 mild and 2 severe/critical cases. There was one case in which both LDL-c and HDL-c levels were $15 \%$ or more reduced at follow-up as compared to the time of admission.

A total of 16 patients ( $n=9$ in mild $n=7$ in severe/ critical cases, Table 1) who had cholesterol-lowering drugs were excluded from the following inter-subgroup analyses. Traditional Chinese medicine is practiced as a regular supplementary treatment to the standard care of Western medicine in China. A substantial portion of patients in this cohort (31 out of 50 in mild group and 17 out of 41 in severe/critical group) had taken either type of TCHMs at home during the onset of their symptoms or / and after discharge; these patients were defined as the TCHM subgroup, whilst the remaining patients were defined as the non-TCHM subgroup. In both mild and severe/critical groups, the median levels of LDL-c, HDLc and non-HDL-c at admission were slightly but insignificantly lower in non-TCHM subgroup as compared with the TCHM subgroup using a Mann-Whitney U test (Table 3). TCHMs also did not cause any significant changes in the levels of LDL-c, HDL-c and non-HDL-c at the time of follow-up in non-TCHM subgroup as compared with TCHM subgroup (Table 3, a MannWhitney U test). In the severe/critical group, however, LDL-c and HDL-c levels in the non-TCHM subgroup were significantly elevated at the time of follow-up as compared to admission, but not in the TCHM subgroup (Fig. 2, Table 3). Less patients in TCHM subgroup took cholesterol-lowering drugs than those in non-TCHM subgroup (Table 3). 
Table 2 Main clinical laboratory values of COVID-19 follow-up patients

\begin{tabular}{|c|c|c|c|c|c|c|c|}
\hline \multirow{3}{*}{$\begin{array}{l}\text { Laboratory } \\
\text { values }\end{array}$} & \multirow{3}{*}{$\begin{array}{l}\text { Reference } \\
\text { ranges }\end{array}$} & \multicolumn{6}{|c|}{ COVID-19 follow-up patients } \\
\hline & & \multicolumn{3}{|l|}{ Mild $(n=59)$} & \multicolumn{3}{|c|}{ Severe/Critical $(n=48)$} \\
\hline & & Adm & Flw & $p$ & Adm & Flw & $p$ \\
\hline LDL-C & *109 (29) mg/dL & $100.5(87.0,113.3)$ & $103.6(90.5,116.0)$ & 0.048 & $96.1(74.3,107.2)$ & $103.6(76.2,123.4)$ & 0.003 \\
\hline $\mathrm{HDL}-\mathrm{C}$ & *52 (15) mg/dL & $52.9(46.0,64.2)$ & $54.5(46.8,63.4)$ & 0.297 & $50.3(43.7,65.4)$ & $55.3(46.0,65.7)$ & 0.042 \\
\hline TC & *182 (34) mg/dL & $197.9(170.5,223.5)$ & $203.4(187.2,220.8)$ & 0.042 & $192.4(151.3,221.6)$ & $203.2(152.1,246.6)$ & 0.016 \\
\hline Non-HDL-C & *130 (35) mg/dL & $144.2(118.3,160.8)$ & $147.0(125.3,165.9)$ & 0.039 & $135.7(102.8,157.4)$ & $151.8(103.7,182.4)$ & 0.009 \\
\hline TG & *133 (99) mg/dL & $165.5(131.1,219.6)$ & $140.8(108.1,177.1)$ & 0.001 & $141.7(107.6,182.2)$ & $143.9(111.6,200.6)$ & 0.376 \\
\hline WBC & $3.5-9.5\left(\times 10^{9} / \mathrm{L}\right)$ & $5.8(5.2,6.8)$ & $5.4(4.7,6.2)$ & 0.002 & $6.3(5.0,8.1)$ & $5.6(5.0,6.7)$ & 0.046 \\
\hline LY & $* 2.1(0.5)\left(\times 10^{9} / \mathrm{L}\right)$ & $1.5(1.3,1.8)$ & $1.9(1.5,2.2)$ & $<0.001$ & $1.6(1.1,1.9)$ & $2.0(1.5,2.3)$ & $<0.001$ \\
\hline MO & $0.1-0.6\left(\times 10^{9} / \mathrm{L}\right)$ & $0.5(0.3,0.6)$ & $0.3(0.2,0.4)$ & $<0.001$ & $0.5(0.3,0.7)$ & $0.3(0.3,0.4)$ & $<0.001$ \\
\hline D-dimer & $<0.5 \mathrm{mg} / \mathrm{ml}$ & $0.5(0.2,0.8)$ & $0.3(0.2,0.4)$ & $<0.001$ & $0.7(0.4,1.1)$ & $0.4(0.3,0.5)$ & $<0.001$ \\
\hline ATIII & $80-120 \%$ & $90(81.5,96.5)$ & $97(89,102.5)$ & $<0.001$ & $88(81.2,93.8)$ & $96(87,102.8)$ & 0.001 \\
\hline FDP & $<5 \mu \mathrm{g} / \mathrm{L}$ & $1.7(1.1,2.5)$ & $1.1(1,1.5)$ & $<0.001$ & $2.7(1.6,3.7)$ & $1.3(1.0,2.0)$ & $<0.001$ \\
\hline FIB & $2-4 \mathrm{~g} / \mathrm{L}$ & $4.0(3.4,5.2)$ & $3.2(2.7,3.5)$ & $<0.001$ & $4.6(3.7,5.5)$ & $3.3(3.0,3.7)$ & $<0.001$ \\
\hline CRP & $<4 \mathrm{mg} / \mathrm{L}$ & $1.7(0.6,4.8)$ & $1.4(0.6,2.2)$ & $<0.001$ & $2.9(1.2,8.3)$ & $1.6(1.1,2.6)$ & 0.002 \\
\hline ALT & $5-35 \cup / L$ & $36(22,54)$ & $25(19,32)$ & $<0.001$ & $33.5(21.8,62.2)$ & $21(15,32.4)$ & 0.013 \\
\hline ALP & 40-150 U/L & $89(72,104)$ & $77(64,87)$ & $<0.001$ & $91(71,107)$ & $73(64,85)$ & $<0.001$ \\
\hline GGT & $7-32 \mathrm{U} / \mathrm{L}$ & $32(22,51)$ & $23(17,32)$ & $<0.001$ & $31.5(18.7,50.5)$ & $23(18,31)$ & $<0.001$ \\
\hline TBIL & $5.1-19 \mu \mathrm{mol} / \mathrm{L}$ & $11.5(9.8,14.6)$ & $16.7(12.2,20.8)$ & $<0.001$ & $11.1(8.8,14.3)$ & $13.2(10.8,15.9)$ & 0.002 \\
\hline DBIL & $1.7-6.8 \mu \mathrm{mol} / \mathrm{L}$ & $3.1(2.5,3.6)$ & $3.7(3,4.5)$ & $<0.001$ & $3.2(2.4,4.0)$ & $3.2(3,3.7)$ & 0.215 \\
\hline TBA & 0-10 $\mu \mathrm{mol} / \mathrm{L}$ & $4.3(3.2,6.9)$ & $2.5(1.5,3.7)$ & $<0.001$ & $4.7(3.3,7.7)$ & $2.6(1.3,4.6)$ & $<0.001$ \\
\hline $\mathrm{TP}$ & $64-83 \mathrm{~g} / \mathrm{L}$ & $70.6(67.6,74.7)$ & $73.8(71.9,77.2)$ & $<0.001$ & $68.9(65.4,72.3)$ & $75.4(72.8,79.5)$ & $<0.001$ \\
\hline GLB & $20-30 \mathrm{~g} / \mathrm{L}$ & $28.6(26.4,31.7)$ & $27.4(24.7,29.7)$ & 0.002 & $28.9(26.4,32.6)$ & $29.0(25.5,31.8)$ & 0.506 \\
\hline ALB & $35-55 \mathrm{~g} / \mathrm{L}$ & $41.9(39.4,44)$ & $46.8(45.7,49)$ & $<0.001$ & $37.4(6.4)$ & $46.5(2.7)$ & $<0.001$ \\
\hline $\mathrm{A} / \mathrm{G}$ ratio & $1.5-2.5$ & $1.5(1.3,1.6)$ & 1. $7(1.5,1.9)$ & $<0.001$ & $1.3(1.2,1.5)$ & $1.6(1.5,1.8)$ & $<0.001$ \\
\hline
\end{tabular}

*Data are adapted from age and gender-matched normal subjects from the city of Wuhan [11]. Data are presented as mean (SD) in reference ranges or median (IQR) unless stated otherwise. A Wilcoxon signed-rank test (two-sided) is used to compare variables at the times of admission with follow-up from the same subjects within groups (mild and severe/critical cases)

Adm at time of admission, Flw follow-up, ATIII antithrombin III, FDP fibrin degradation product, FIB fibrinogen, $A L T$ alanine aminotransferase, $A L P$ alkaline phosphatase, GGT gamma-glutamyl transferase, $W B C$ white blood cell, $L Y$ lymphocyte, $M O$ monocyte, $T C$ total cholesterol, $T G$ triglycerides, $T P$ total protein, $A L B$ albumin, GLB globulin, TBIL total bilirubin, DBIL direct bilirubin, CRP C-reactive protein, TBA total bile acid, A/G ALB/GLB ratio

\section{Improvements of other serum laboratory values in recovered COVID-19 patients}

We further compared levels of other serum laboratory values at 3-6 months after discharge to those at the time of admission. Levels of numerous physiopathological markers showed significant improvements in patients at follow-up as compared to the time of admission across all subgroups; these laboratory values included LY, WBC, ALP, ALT, GGT, MO, CRP, and ALB, coagulation markers such as D-dimer, ATIII, FDP, and FIB (Table 2).

\section{Relationships of ratios of WBC and ratios of HDL-C}

We performed a correlation analysis to calculate the correlation coefficiency between ratios of LDL-c or HDL-c and ratios of some laboratory values from follow-up to the time of admission after exclusion of subjects taking cholesterol-lowering drugs. Increases in HDL-c significantly correlated with increases in numbers of WBC $(\mathrm{R}=0.336, p=0.001)$ (Fig. 2). In addition, changes in levels of LDL-c or HDL-c both significantly correlated with changes in levels of ALP (LDL-c, $R=0.1260, p=0.014$; HDL-c, $R=0.352, p<0.001$ ), ALT (LDL-c, $R=0.271, p=$ 0.011 ; HDL-c, $R=0.239, p=0.025)$, TP (LDL-c, $R=0.424$, $p<0.001$; HDL-c, $R=0.228, p=0.032$ ) and ALB (LDL-c, $R=0.402, p<0.001 ;$ HDL-c, $R=0.323, p=0.002)$.

\section{Incomplete resolution of lung lesions in $\mathrm{CT}$ findings}

A total of 61 of 107 patients had a revisit CT examination at follow-up; these patients included 31 mild, 24 severe, and 6 critical cases. The main types of abnormalities in CT images include ground glass opacities (GGO), consolidation/nodules, and shadows. GGO and large diffuse shadows were two typical patterns of abnormalities found in CT images at the time of admission, suggesting diffuse 


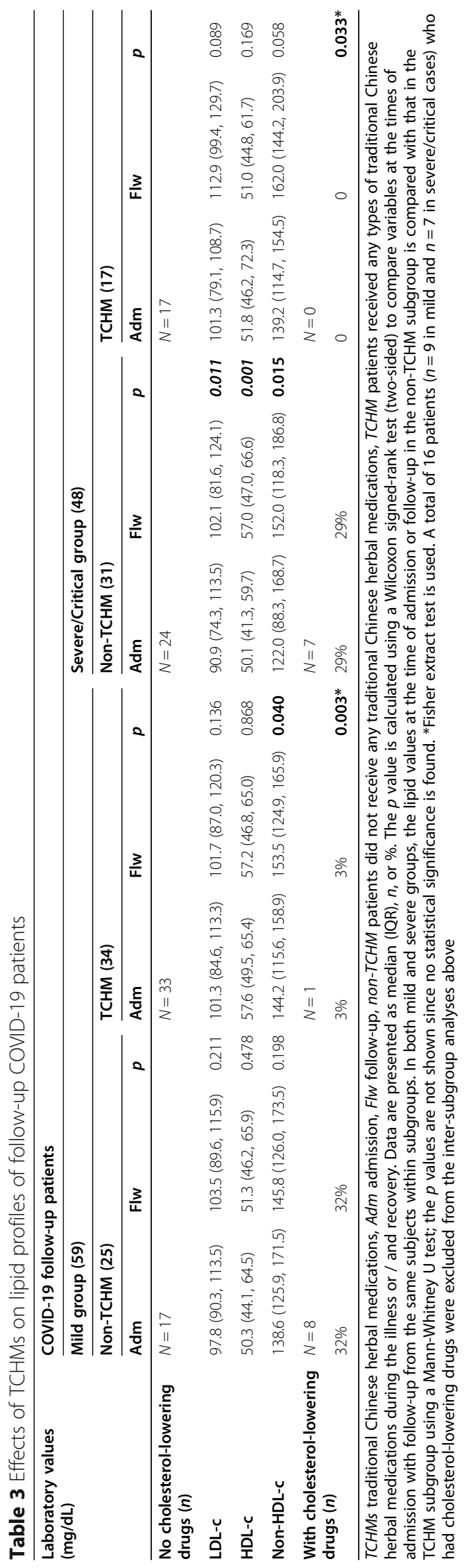



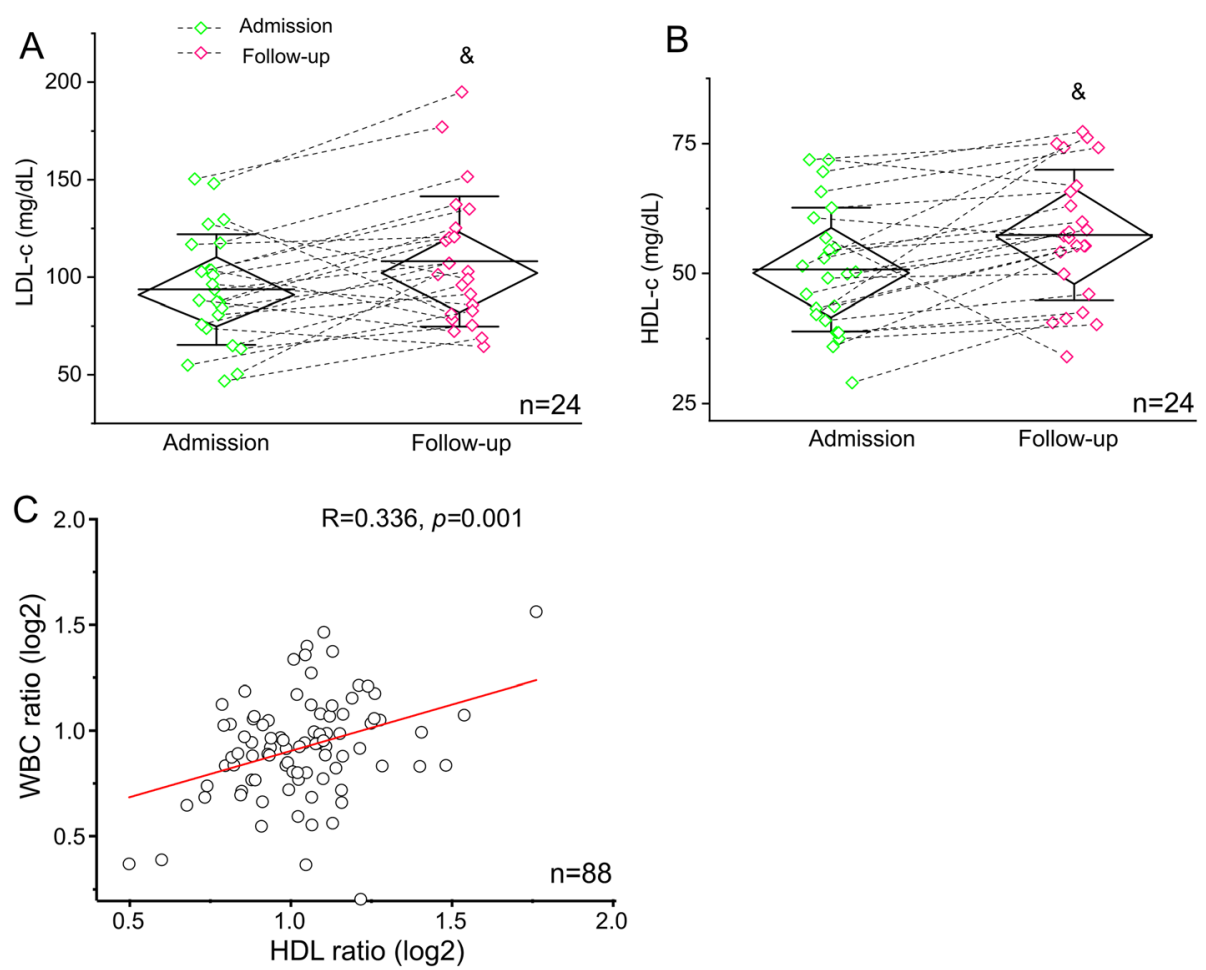

Fig. 2 Restoration of lipid levels and correlations of ratio of WBC and ratio of HDL-c levels in recovered COVID-19 patients. Serum LDL-c a and $\mathrm{HDL}-\mathrm{c} \mathbf{b}$ levels are presented from COVID-19 patients in non-TCHM severe/critical group without taking cholesterol-lowering drugs $(n=24)$ at follow-up and the time of admission. Diamond boxes represent median (IQR) and whiskers represent SD in the plots. Dotted lines connect data points at the time of admission and at follow-up from the same subjects. A Wilcoxon signed-rank test (two-sided) is used to compare the variables at the times of admission with follow-up from the same subjects within subgroups. Note: " $\&$ " indicates statistically significant. c Correlations of ratio of WBC and ratio of HDL-c levels at the time of follow-up to admission in the follow-up COVID-19 patients. Increases in HDLc significantly correlate with increases in numbers of WBC (in log2 scale). A Spearman correlation analysis is used. WBC, white blood cells

and acute lung inflammations. In follow-up CT images, consolidations/nodules and small scattered shadows were the main types of residue lesions, suggesting improvements and incomplete absorbing process during recovery. A total of $29 \%$ of patients (17 of 61 ) showed a complete resolution of lung lesions in follow-up CT images. A total of $72 \%$ of patients (44 of 61 ) showed incomplete resolution, that is, residue lesions, in follow-up CT images as compared with CT findings at the time of admission. We did not find any significant correlations between cholesterol levels and residual lesions (data not shown).

\section{Discussion}

In this study, we performed a follow-up investigation of lipid profiles and other laboratory values on 107 recovered COVID-19 patients at 3-6 months after discharge. Our data demonstrate that levels of LDL-c and HDL-c increased significantly in severe/critical COVID-19 cases with or without adjustment of the application of traditional Chinese medicine. Coagulation and liver laboratory values, including D-dimer, ATIII, FDP, FIB, CRP, ALT, ALP, and GGT, decreased significantly across all subgroups. Furthermore, incomplete absorption of lung lesions was observed in $\mathrm{CT}$ images in most follow-up patients. These findings provide insight into the pathological evolution of COVID-19 during recovery and potential long-term sequelae of the disease.

Recently, we and other investigators have reported hypolipidemia in hospitalized COVID-19 patients [10-13]. The decrease in lipid levels in patients with COVID-19 is associated with the severity of the symptoms [10-12]. These findings demonstrate that abnormalities in lipid metabolism are clinical manifestations of COVID-19 that have been underappreciated. Mild or moderate liver injuries caused by viral infection may be one important factor contributing to dyslipidemia in COVID-19 patients. Serum levels of ALT, ALP, and GGT were moderately elevated in about half of the cohort of patients in our study at the time of admission, indicating mild or moderate liver injury [11]. In this study, patient ALT, ALP, and GGT levels were significantly lower at follow-up than at the time of admission, indicating improvements in liver enzyme levels in patients during recovery. There are a couple of potential mechanisms involved in the role of cholesterol in the pathological progression of COVID-19. Wang et al. suggests that cholesterol concomitantly traffics ACE2 to viral entry sites, where 
SARS-CoV-2 docks in order to properly exploit entry into cells [19]. Therefore, decreased cholesterol levels in the blood may indicate severe loading of cholesterol in peripheral tissue and escalated SARS-CoV-2 infectivity [19]. Cao et al. suggests that cholesterol may facilitate an acceleration of endothelial injuries caused by SARS-CoV-2 [20]. Sorokin suggests that lowering HDL-c in COVID-19 patients may decrease the anti-inflammatory and antioxidative functions of HDL-c and contribute to pulmonary inflammation [13]. The decrease in WBC and increase in lymphocytes at follow-up may be reflected cytokine secretion and inflammatory status and related to lipid parameters. In addition, serum amyloid A protein (SAA) level can rise up to 1000fold in response to an acute inflammation, which will displace apolipoprotein A-I from HDL particles [21]. HDL containing SAA is targeted to the macrophage [22]. SAA level increases in COVID-19 patients, especially severe cases $[23,24]$. It is likely that SAA/apolipoprotein A-I axis gets involved in HDL-c metabolism in COVID-19 patients, but the detailed mechanism needs to be elucidated in future studies. All these hypotheses will lead to more and novel insights into the nature of this disease.

The dynamics of lipid levels in a small cohort of our longitudinal study and in two cases in other reports have shown that cholesterol levels were low at the time the patients were hospitalized, remained low during disease progression, and returned to baseline levels in patients who were discharged $[10,12,13]$. To our surprise, after exclusion of the factor of taking cholesterol-lowering drugs, a small portion of patients (6\%) showed a decrease in LDL-c or HDL-c levels of $15 \%$ or more at follow-up as compared to the time of admission. The low lipid levels in these patients were probably due to medications or nutritional supplements taken during their own recovery process at home, for example, profound and acute dietary changes. It would be interesting to find out whether those patients with lower LDL-c or HDL-c levels at follow-up were associated with malnutritional or socioeconomically underrepresented populations. Loss of appetite is one of early symptoms for some COVID-19 patients. They may continue having a poor appetite during the disease progression and recovery courses which may result in malnutrition thus low lipid levels at follow-up. Low lipid levels may also be caused by the matter of patient compliance after discharge. Although it is less likely, there could be a longterm sequela of lipid abnormality caused by or associated with viral infection in COVID-19 patients; there is no evidence to support the notion that SARS-CoV-2 causes long-term chronic infection.

Emerging evidence has supported coagulation as an independent mortality factor in COVID-19 patients. Coagulopathies have been found in the early stages of the disease [25-27] and in non-surviving patients
[28]. Patients have shown elevated coagulation and cardiac biomarkers such as D-dimer, fibrinogen, highsensitivity troponin I and creatinine kinase-myocardial band $[29,30]$. In our follow-up study, coagulation laboratory values, including D-dimer, ATIII, FDP, and FIB, were significantly lower in patients at follow-up as compared to the time of admission across all subgroups, indicating improvements from coagulopathies. However, we did not find significant correlations between the restoration of LDL-c or HDL-c levels and decreases in levels of these coagulation values; this suggests that recovery from dyslipidemia and improvements from coagulopathies are probably involving different pathways at different paces.

Incomplete resolution of lung lesions was observed in $72 \%$ patients in the follow-up CT examinations, suggesting pulmonary fibrosis as a potential long-term sequela for many COVID-19 patients. SARS patients have shown persistent impairment of lung function, even years after discharge [31, 32]. Pulmonary fibrosis, GGO, and pleural thickening have been reported in follow-up chest radiographs in a substantial portion of patients with Middle East respiratory syndrome coronavirus (MERS-CoV) [33]. Consistent with our findings, You et al. showed that $83.3 \%$ of COVID-19 patients had residual CT abnormalities, including GGO and pulmonary fibrosis [34]. These data suggest that aberrant wound healing in COVID-19 survivors, which is evidenced by GGO and residue lesion patterns, may lead to pulmonary fibrosis; larger studies are needed to verify this notion.

There were several limitations of this study. First, less than one-fifth of the patients from our original cohort participated in this study, which might cause a biased representative sample group from the original cohort. Second, the sample size for follow-up critical cases was limited; this might lead to an overall insignificant increase in levels of LDL-c in this subgroup. Third, many patients might have been taking various medications or remedies at home for recovery, including Chinese traditional medicines or nutritional supplements. In this study, we found that about $58 \%$ of patients in mild group and $37 \%$ of patients in severe group had taken TCHMs during their illness or / and recovery courses. Our data indicated that TCHMs might have a negative impact on the improvement of lipid profiles in patients with severe symptoms. However, due to the complexity of ingredients in those TCHMs, it will be very difficult to determine which factor(s) and how they interfere with lipid metabolisms in some patients' recoveries in the severe group; this will need a thorough investigation in future. We, however, did not find so far that TCHMs caused any significant changes in the overall lipid profiles at the time of admission and follow-up crossing all the subgroups. Therefore, TCHMs might have a minor 
effect on lipid values in our patients which resulted in a negligible impact on the conclusions we drew in this study. We are aware that these data and analyses only apply to this specific Chinese population. Fourth, the lipid profiles of patients prior to discharge were crucial to determine the contributive factors to the decreased LDL-c or HDL-c levels in a small portion of patients at follow-up as compared to admission, which were lacking. Fifth, a continuous long-term follow-up is needed in order to monitor the dynamics of lipid profiles and CT abnormalities during the recovery process for a large cohort of COVID-19 patients in order to better predict potential sequelae, such as lung fibrosis; this will be our future research goal. Lastly, the characteristics of lipoproteins, such as apolipoprotein A-I, in our cohort was unknown. We also did not know the cellular cholesterol levels in COVID-19 in this study; such information could provide us insights into the molecular mechanisms underlying dyslipidemia in COVID-19. Whether and how cholesterol or lipoproteins participate in regulation of SARS-CoV-2 entry of host cells and viral production are yet to be determined, which will our primary goal in future investigations.

\section{Conclusion}

Collectively, our data show improvements of LDL-c and HDL-c and incomplete absorption of lung lesions in COVID-19 patients at a 3-6-month follow-up, indicating a long-term recovery process and the development of potential sequelae such as pulmonary fibrosis.

\section{Abbreviations \\ COVID-19: Coronavirus disease 2019; SARS-Cov-2: Severe acute respiratory syndrome coronavirus 2; ACE2: Angiotensin-converting enzyme 2; TMPR SS2: Transmembrane, serine protease 2; SARS: Severe acute respiratory syndrome; ATIII: Antithrombin III; FDP: Fibrin degradation product; FIB: Fibrinogen; ALT: Alanine aminotransferase; ALP: Alkaline phosphatase; GGT: Gamma-glutamyl transferase; LDL-c: Low-density lipoprotein cholesterol; TCHM: Traditional Chinese herbal medications; WBC: White blood cell; LY: Lymphocyte; MO: Monocyte; HDL-c: High-density lipoprotein cholesterol TC: Total cholesterol; TG: Triglycerides; TP: Total protein; ALB: Albumin; GLB: Globulin; TBIL: Total bilirubin; DBIL: Direct bilirubin; TBA: Total bile acid; CRP: C-reactive protein; GGO: Ground glass opacities; IQR: Interquartile range}

\section{Acknowledgements}

The authors are very grateful to the assistance and help from Dr. Rong Yin at the Department of Cell Biology and Anatomy School of Medicine University of South Carolina for the data analysis.

\section{Prior publication}

None of the material in this manuscript has been published or is under consideration for publication elsewhere, including the Internet and conferences.

\section{Authors' contributions}

GL, LD, HW, and WT supervised and designed the study. GL, LD, XW, YJ, YL, and HW performed the tests and collected the data. XC, VG, and WT performed data analysis and interpretation. WT wrote and revised the manuscript. The authors read and approved the final manuscript.

\section{Funding}

This study was supported by the Health and Family Planning Commission of Hubei Province (Grant No. WJ2019M158 to HW).

\section{Availability of data and materials}

The collection of data that supports the findings in this study is available from the Union Hospital, Wuhan, but restrictions may apply to the availability of these data, which were used under license for the current study, and so are not publicly available. Data are however available from the authors upon reasonable request and with permission of the Union Hospital, Wuhan.

\section{Declarations}

Ethics approval and consent to participate

This study was approved by the Institutional Review Board (IRB) at the Union Hospital of Tongji Medical College, Huazhong University of Science and Technology in Wuhan, P. R. China. Oral consent was obtained from the participants. The need for written informed consent was waived by the IRB committee at the Union Hospital of Tongji Medical College, Huazhong University of Science and Technology in Wuhan, P. R. China.

\section{Consent for publication}

Not applicable.

\section{Competing interests}

The authors do not have any professional and financial affiliations that may be perceived to have biased the presentation.

\section{Author details}

${ }^{1}$ Cancer Center, Union Hospital, Tongji Medical College, Huazhong University of Science and Technology, Wuhan 430022, People's Republic of China. 2Division of Gastroenterology, Union Hospital, Tongji Medical College, Huazhong University of Science and Technology, Wuhan 430022, People's Republic of China. ${ }^{3}$ Department of Cell Biology and Anatomy, School of Medicine, University of South Carolina, Columbia, SC 29209, USA.

${ }^{4}$ Department of Laboratory Medicine, Cancer Center, Union Hospital, Tongji Medical College, Huazhong University of Science and Technology, Wuhan 430022, People's Republic of China. ${ }^{5}$ Department of Biomedical Engineering, College of Engineering and Computing, University of South Carolina, Columbia, SC, USA. ${ }^{6}$ Department of Laboratory Medicine, Union Hospital, Tongji Medical College, Huazhong University of Science and Technology, Wuhan 430022, People's Republic of China.

Received: 11 January 2021 Accepted: 9 March 2021

Published online: 24 March 2021

\section{References}

1. Wu Z, McGoogan JM. Characteristics of and important lessons from the coronavirus disease 2019 (COVID-19) outbreak in China: summary of a report of 72314 cases from the Chinese Center for Disease Control and Prevention. JAMA. 2020;323(13):1239-42. https://doi.org/1 0.1001/jama.2020.2648

2. Xu X, Chen P, Wang J, Feng J, Zhou H, Li X, Zhong W, Hao P. Evolution of the novel coronavirus from the ongoing Wuhan outbreak and modeling of its spike protein for risk of human transmission. Sci China Life Sci. 2020; 63(3):457-60. https://doi.org/10.1007/s11427-020-1637-5.

3. COVID-19 Dashboard by the Center for Systems Science and Engineering (CSSE) at Johns Hopkins University (JHU). In., 2020 edn: ArcGIS. Johns Hopkins University; 2020.

4. Lu R, Zhao X, Li J, Niu P, Yang B, Wu H, Wang W, Song H, Huang B, Zhu N, Bi Y, Ma X, Zhan F, Wang L, Hu T, Zhou H, Hu Z, Zhou W, Zhao L, Chen J, Meng Y, Wang J, Lin Y, Yuan J, Xie Z, Ma J, Liu WJ, Wang D, Xu W, Holmes EC, Gao GF, Wu G, Chen W, Shi W, Tan W, et al. Genomic characterisation and epidemiology of 2019 novel coronavirus: implications for virus origins and receptor binding. Lancet. 2020;395(10224):565-74. https://doi.org/10.1 016/S0140-6736(20)30251-8.

5. Wan Y, Shang J, Graham R, Baric RS, Li F. Receptor recognition by novel coronavirus from Wuhan: an analysis based on decade-long structural studies of SARS. J Virol. 2020;94(7). https://doi.org/10.1128/JVI.00127-20.

6. Lauer SA Grantz KH, Bi O Jones FK, Zheng O Meredith HR, Azman AS, Reich NG, Lessler J. The incubation period of coronavirus disease 2019 
(COVID-19) from publicly reported confirmed cases: estimation and application. Ann Intern Med. 2020;172(9):577-82. https://doi.org/10.7326/M2 0-0504.

7. Cao WJ, Wang TT, Gao YF, Wang YQ, Bao T, Zou GZ. Serum Lipid Metabolic Derangement is Associated with Disease Progression During Chronic HBV Infection. Clin Lab. 2019;65:12.

8. Wei X, Su J, Yang K, Wei J, Wan H, Cao X, Tan W, Wang H. Elevations of serum cancer biomarkers correlate with severity of COVID-19. J Med Virol. 2020;92(10):2036-41. https://doi.org/10.1002/jmv.25957.

9. Zhang P, Li J, Liu H, Han N, Ju J, Kou Y, Chen L, Jiang M, Pan F, Zheng Y, Gao Z, Jiang B, et al. Long-term bone and lung consequences associated with hospital-acquired severe acute respiratory syndrome: a 15-year followup from a prospective cohort study. Bone Res. 2020;8(1):8. https://doi.org/1 0.1038/s41413-020-0084-5.

10. Fan J, Wang H, Ye G, Cao X, Xu X, Tan W, Zhang Y. Low-density lipoprotein is a potential predictor of poor prognosis in patients with coronavirus disease 2019. Metabolism. 2020:154243.

11. Wei X, Zeng W, Su J, Wan H, Yu X, Cao X, Tan W, Wang H. Hypolipidemia is associated with the severity of COVID-19. J Clin Lipidol. 2020;14(3):297-304. https://doi.org/10.1016/j.jacl.2020.04.008.

12. Hu X, Chen D, Wu L, He G, Ye W. Declined serum high density lipoprotein cholesterol is associated with the severity of COVID-19 infection. Clin Chim Acta. 2020;510:105-10. https://doi.org/10.1016/j.cca.2020.07.015.

13. Sorokin AV, Karathanasis SK, Yang ZH, Freeman L, Kotani K, Remaley AT. COVID-19-associated dyslipidemia: implications for mechanism of impaired resolution and novel therapeutic approaches. FASEB J. 2020;34(8):9843-53. https://doi.org/10.1096/fj.202001451.

14. Coronavirus COVID-19 (SARS-CoV-2), Johns Hopkins ABX Guide [https:// www.hopkinsguides.com/hopkins/view/Johns_Hopkins_ABX_Guide/54074 7/all/Coronavirus_COVID_19_SARS_COV_2].

15. Song SZ, Liu HY, Shen H, Yuan B, Dong ZN, Jia XW, Tian YP. Comparison of serum biochemical features between SARS and other viral pneumonias. Zhongguo Wei Zhong Bing Ji Jiu Yi Xue. 2004;16(11):664-6.

16. Wu Q, Zhou L, Sun X, Yan Z, Hu C, Wu J, Xu L, Li X, Liu H, Yin P, Li K, Zhao J, Li Y, Wang X, Li Y, Zhang Q, Xu G, Chen H, et al. Altered lipid metabolism in recovered SARS patients twelve years after infection. Sci Rep. 2017;7(1):9110. https://doi.org/10.1038/s41598-017-09536-z.

17. Wongjitrat $C$, Sukwit $S$, Chuenchitra T, Seangjaruk $P$, Rojanasang $P$, Romputtan P, Srisurapanon S. CTLA-4 and its ligands on the surface of Tand B-lymphocyte subsets in chronic hepatitis B virus infection. J Med Assoc Thail. 2013;96(Suppl 1):S54-9.

18. Shi H, Han X, Jiang N, Cao Y, Alwalid O, Gu J, Fan Y, Zheng C. Radiological findings from 81 patients with COVID-19 pneumonia in Wuhan, China: a descriptive study. Lancet Infect Dis. 2020;20(4):425-34. https://doi.org/10.101 6/S1473-3099(20)30086-4.

19. Wang H, Yuan Z, Pavel MA, Hansen SB. The role of high cholesterol in agerelated COVID19 lethality. bioRxiv. 2020.

20. Cao X, Yin R, Albrecht H, Fan D, Tan W. Cholesterol: a new game player accelerating vasculopathy caused by SARS-CoV-2? Am J Physiol Endocrinol Metab. 2020;319(1):E197-202. https://doi.org/10.1152/ajpendo.00255.2020.

21. Eklund KK, Niemi K, Kovanen PT. Immune functions of serum amyloid a. Crit Rev Immunol. 2012;32(4):335-48. https://doi.org/10.1615/CritRevImmunol. v32.14.40.

22. Sack GH Jr. Serum amyloid a (SAA) proteins. Subcell Biochem. 2020;94:42136. https://doi.org/10.1007/978-3-030-41769-7_17.

23. Goncalves CA, Sesterheim P. Serum amyloid a protein has been undervalued as a biomarker of COVID-19. Diabetes Metab Res Rev. 2021; 37(1):e3376. https://doi.org/10.1002/dmrr.3376.

24. Haroun RA, Osman WH, Eessa AM. Interferon-gamma-induced protein 10 (IP-10) and serum amyloid a (SAA) are excellent biomarkers for the prediction of COVID-19 progression and severity. Life Sci. 2021;269:119019. https://doi.org/10.1016/j.lfs.2021.119019.

25. Lodigiani C, lapichino G, Carenzo L, Cecconi M, Ferrazzi P, Sebastian T, Kucher N, Studt JD, Sacco C, Alexia B, et al. Venous and arterial thromboembolic complications in COVID-19 patients admitted to an academic hospital in Milan, Italy. Thromb Res. 2020;191:9-14. https://doi. org/10.1016/j.thromres.2020.04.024.

26. Klok FA, Kruip M, van der Meer NJM, Arbous MS, Gommers D, Kant KM, Kaptein FHJ, van Paassen J, Stals MAM, Huisman MV, et al. Incidence of thrombotic complications in critically ill ICU patients with COVID-19. Thromb Res. 2020.
27. Helms J, Tacquard C, Severac F, Leonard-Lorant I, Ohana M, Delabranche X, Merdji H, Clere-Jehl R, Schenck M, Fagot Gandet F, et al. High risk of thrombosis in patients with severe SARS-CoV-2 infection: a multicenter prospective cohort study. Intensive Care Med. 2020:1-10.

28. Tang N, Li D, Wang X, Sun Z. Abnormal coagulation parameters are associated with poor prognosis in patients with novel coronavirus pneumonia. J Thromb Haemost. 2020;18(4):844-7. https://doi.org/10.1111/ jth.14768.

29. Shi S, Qin M, Shen B, Cai Y, Liu T, Yang F, Gong W, Liu X, Liang J, Zhao Q et al: Association of Cardiac Injury with Mortality in hospitalized patients with COVID-19 in Wuhan, China. JAMA Cardiol 2020, Association of Cardiac Injury With Mortality in Hospitalized Patients With COVID-19 in Wuhan, China.

30. Gao Y, Li T, Han M, Li X, Wu D, Xu Y, Zhu Y, Liu Y, Wang X, Wang L. Diagnostic utility of clinical laboratory data determinations for patients with the severe COVID-19. J Med Virol. 2020;92(7):791-6. https://doi.org/10.1002/ jmv.25770.

31. Xie L, Liu Y, Xiao Y, Tian Q, Fan B, Zhao H, Chen W. Follow-up study on pulmonary function and lung radiographic changes in rehabilitating severe acute respiratory syndrome patients after discharge. Chest. 2005;127(6): 2119-24. https://doi.org/10.1378/chest.127.6.2119.

32. Hui DS, Wong KT, Ko FW, Tam LS, Chan DP, Woo J, Sung JJ. The 1-year impact of severe acute respiratory syndrome on pulmonary function, exercise capacity, and quality of life in a cohort of survivors. Chest. 2005; 128(4):2247-61. https://doi.org/10.1378/chest.128.4.2247.

33. Das KM, Lee EY, Singh R, Enani MA, Al Dossari K, Van Gorkom K, Larsson SG, Langer RD. Follow-up chest radiographic findings in patients with MERSCoV after recovery. Indian J Radiol Imag. 2017;27(3):342-9. https://doi.org/1 0.4103/ijri.IJRI_469_16.

34. You J, Zhang L, Ni-Jia-Ti MY, Zhang J, Hu F, Chen L, Dong Y, Yang K, Zhang $B$, Zhang $S$. Anormal pulmonary function and residual $C T$ abnormalities in rehabilitating COVID-19 patients after discharge. J Inf Secur. 2020.

\section{Publisher's Note}

Springer Nature remains neutral with regard to jurisdictional claims in published maps and institutional affiliations.
Ready to submit your research? Choose BMC and benefit from:

- fast, convenient online submission

- thorough peer review by experienced researchers in your field

- rapid publication on acceptance

- support for research data, including large and complex data types

- gold Open Access which fosters wider collaboration and increased citations

- maximum visibility for your research: over $100 \mathrm{M}$ website views per year

At BMC, research is always in progress.

Learn more biomedcentral.com/submissions 